\title{
A Horizontal Brazil-Nut Effect and Its Reverse
}

\author{
T. Schnautz, ${ }^{1}$ R. Brito, ${ }^{2}$ C. A. Kruelle, ${ }^{1}$ and I. Rehberg ${ }^{1}$ \\ ${ }^{1}$ Experimentalphysik V, Universität Bayreuth, D-95440 Bayreuth, Germany \\ ${ }^{2}$ Dpt. de Física Aplicada I and GISC, Universidad Complutense, E-28040 Madrid, Spain
}

(Received 7 December 2004; published 7 July 2005)

\begin{abstract}
Transport effects in a monolayer consisting of a binary granular mixture, confined in a horizontally vibrating circular dish, are studied experimentally and compared with a reduced theoretical model. Depending on the ratio of the particles' material density and size, migration of the larger particles occurs either towards the boundary or to the center of the circular container. These directed motions show similarities to the Brazil-nut effect and its reverse form.
\end{abstract}

DOI: 10.1103/PhysRevLett.95.028001

PACS numbers: $45.70 . \mathrm{Mg}, 05.20 .-\mathrm{y}$

The gold miners at the Klondike river gold rush of 1899 knew that by applying a swirling motion to a pan of glittering sand the desired gold nuggets were slowly exposed [1]. Such segregation effects of granular mixtures are of rising interest for scientists and industrial engineers and have been the subject of considerable study in the engineering [2] and physics community [3] during the last decades. When granular materials are mechanically agitated, particles of different sizes, density, or other physical properties tend to separate. This unmixing of ingredients is a well-known nuisance, e.g., in the pharmaceutical industry, where a controlled and homogeneous mixture of powdered drugs with binder is highly desirable.

Several segregation mechanisms have been proposed, including sifting (percolation of small particles through gaps between large particles) [4,5], local rearrangements (large particles being pushed upwards by smaller grains entering into voids beneath them) [6-9], and convection (entrainment of large particles in a convective flow) [10]. These segregation effects have been investigated recently in different experimental setups like rotating drums, granular flows, and vertically or horizontally vibrated systems (for a recent review, see [3]).

Most reports are about vertically shaken granular mixtures, where the larger particles will end up on top of the smaller ones. This so-called "Brazil-nut effect" has attracted a lot of attention in the last years $[8,10,11]$. Moreover, it came as a surprise when Shinbrot and Muzzio [12] observed that a large particle could also sink to the bottom of the container if the bed was deep and the amplitude of vibration large. This puzzling phenomenon has become known as "reverse Brazil-nut effect." Recent theoretical and experimental studies [13-19] have investigated under which conditions both effects, the rise or descent of the larger particles, may occur.

In addition to the vertically vibrated systems, in the last years, some works have also focused on horizontally driven systems. For example, the granular clustering in a horizontally shaken binary layer of dry particles has been investigated by Aumaitre et al. [20,21] and Reis et al. [22,23].
The clustering phenomena were explained by the concept of depletion forces.

In this Letter we report on a new type of granular transport mechanism devoid of depletion forces by utilizing just one single intruder particle, embedded in a monolayer of glass beads. The intruder migrates either to the center or to the boundary of the swirled container, depending on both the size and the material density. This is illustrated for a collection of arbitrarily chosen intruder particles in Fig. 1. For this figure we have used nine particles - unlike in the rest of the Letter-in order to demonstrate that these effects show a striking resemblance to the crossover be-

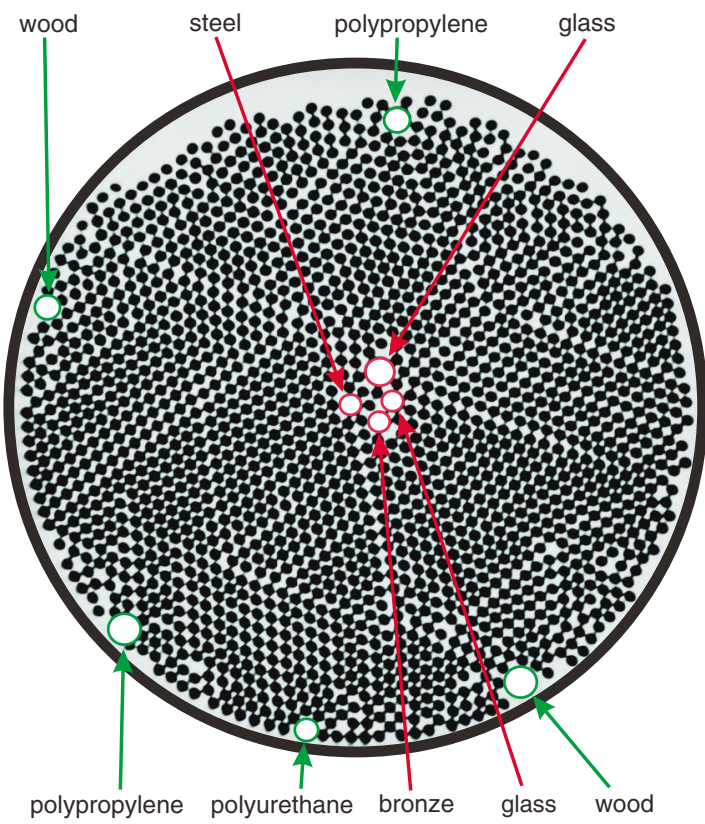

FIG. 1 (color). Snapshot of 1600 glass spheres (diameter $d=$ $6 \mathrm{~mm}$, filling fraction $\mu=0.68$ ) in a swirling dish of diameter $D=29 \mathrm{~cm}$. After several minutes of horizontal swirling agitation, nine selected intruder particles made of various materials with different sizes are driven either to the center of the container or to its boundary. Shaker amplitude $A=1.91 \mathrm{~cm}$ and frequency $f=1.67 \mathrm{~Hz}$. 
tween the Brazil-nut effect and its reverse in vertically agitated systems [16].

The term "swirling" is used for describing a horizontal circular vibration, i.e., a circular movement of the entire platform due to the superposition of two sinusoidal vibrations in perpendicular directions. During one oscillation period every point on the table moves on a circle in the laboratory frame, with an amplitude $A$ of the orbital motion and driving frequency $f$ of the shaker. It is important to note that this circular motion has no center of rotation. The frequency $f$ of the table motion can be tuned from 0.5 to $2.0 \mathrm{~Hz}$, with a preset amplitude $A=\frac{n}{8} \cdot 2.54 \mathrm{~cm}$, where $n=3, \ldots, 7$. During the motion, the positions of all particles are captured with a charge-coupled-device (CCD) camera mounted on top of the swirling table. By this, the experiment is monitored in the comoving frame. The images can be analyzed in real time by computerized imageprocessing algorithms as well as stored on hard disk for further evaluations.

In our experiments we load the dish of diameter $D=$ $29 \mathrm{~cm}$ with a partially filled monolayer of glass beads. One single particle of different size and/or material density is added at a distance $D / 4$ from the center of the container. When the oscillation starts the sphere either tends to move towards the center or to the boundary of the container. In few cases the intruder particle did not show any sign of net motion. After having obtained an idea of the larger sphere's direction of movement, the initial conditions for the measurement are changed: spheres with the tendency to segregate to the center of the container were placed close to the boundary wall, and spheres known to be moving towards the boundary wall were placed in the center of the container. When the oscillation was started, the position of the larger sphere was captured by the CCD camera in constant time steps of $0.05 \mathrm{sec}$.

The top part of Fig. 2 shows the temporal evolution of the radial distance $d_{\mathrm{p}}$ of a $10 \mathrm{~mm}$ intruder polypropylene sphere, which is surrounded by $6 \mathrm{~mm}$ glass beads. It starts at the center of the container and edges through the bulk of the small particles towards the cluster's boundary, where it stays. The remaining oscillations are due to the swirling motion of the dish that induces cycloidal motions of the particles. Experimental data are indicated by the green line, while the corresponding numerical results, to be described below, are plotted in black.

In contrast, the lower part of Fig. 2 demonstrates the reverse effect: a $10 \mathrm{~mm}$ steel ball initially placed at the boundary is driven towards the central region of the cluster. Therefore, by changing the intruders' density, we have reversed the direction of migration. This behavior resembles the crossover between the Brazil-nut and reverse Brazil-nut effect.

In order to check whether these effects are reproducible and to smear out the fluctuations, the experiments were repeated 10 times. Figure 3 shows the temporal evolution

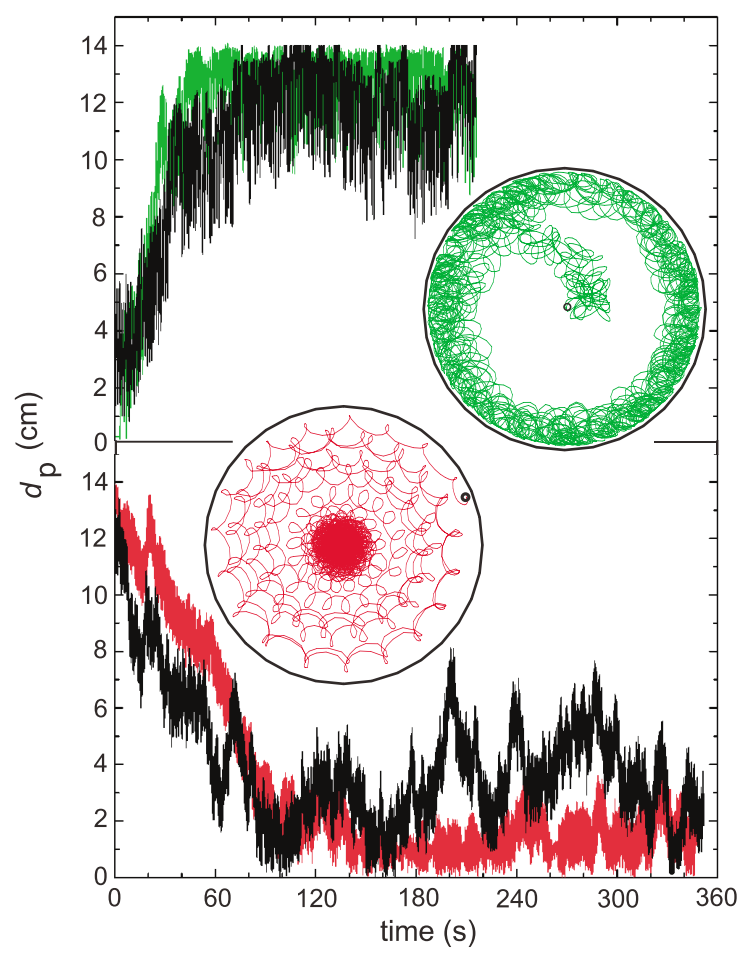

FIG. 2 (color). Temporal evolution of the radial distance $d_{\mathrm{p}}$ from the center of the swirling dish for two different intruder particles. Top: $10 \mathrm{~mm}$ polypropylene sphere in 1500 glass beads ( $d=6 \mathrm{~mm}$, filling fraction $\mu=0.64$ ) migrating to the boundary of the container. Bottom: $10 \mathrm{~mm}$ steel ball in 1800 glass beads (filling fraction $\mu=0.77$ ) being driven to the center of the dish. Black solid lines indicate the results of complementary numerical simulations. The two insets show the corresponding experimental traces of the intruder particles in a comoving frame, with the initial starting positions marked by black circles. Shaker amplitude $A=1.59 \mathrm{~cm}$ (top) and $A=2.22 \mathrm{~cm}$ (bottom), and frequency $f=1.67 \mathrm{~Hz}$ in both cases.

of the averaged distance $d_{\mathrm{p}}$ for both particle species discussed above. A characteristic time, $t_{0}$, can be defined by an exponential fit to the experimental data. For the data shown in Fig. 3 we obtain $t_{0}=(37.7 \pm 0.7) \mathrm{s}$ for migration to the boundary and $t_{0}=(34.7 \pm 0.6) \mathrm{s}$ towards the center.

Complementary to experimental investigation we performed computer simulations of a theoretical model in order to pinpoint the relevant parameters driving the migration. We use an "event-driven" (ED) code adapted to describe a swirling dish filled with $N$ hard spheres of various masses $m_{i}$ and diameters $d_{i}$. As the acceleration of the dish is relatively small, we can assume that the particles do not slide but roll with velocity $\vec{v}$ on the dish's surface. This condition leads to an acceleration of the spheres which is $2 / 7$ times the acceleration of the swirling dish [24]. Moreover, we neglect the spin of the particles apart of that related with the rolling condition, namely $\vec{\omega}=$ $\frac{\vec{\sigma} \times \vec{v}}{|\vec{\sigma}|^{2}}$, where $\vec{\sigma}$ is the vector pointing from the center of the 


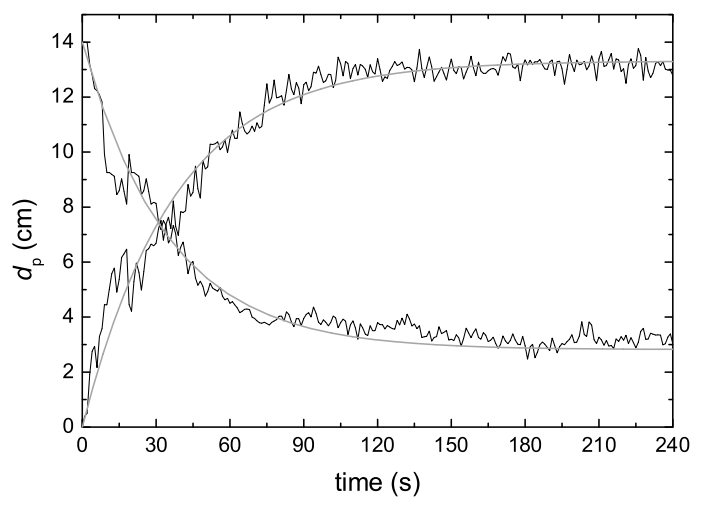

FIG. 3. Temporal evolution of the radial distance $d_{\mathrm{p}}$ for two different intruder particles averaged over 10 runs, together with exponential fits. Experimental details as specified in Fig. 2.

particle to its contact point with the base. In addition, we include a friction force $F_{i}=\mu_{r} m_{i} g$ opposing the direction of motion, where $g$ is the acceleration of gravity. We take a realistic value of $\mu_{r}=0.002$ that represents the mean value for the different materials used in the experiments.

The collisions between particles are inelastic characterized only by a normal restitution coefficient $\alpha$. We set $\alpha=$ 0.86 , which is the average value of our experimental measurements for glass beads. We do not take into account a tangential coefficient of restitution, as it typically has no measurable impact [11]. Collisions of particles with the wall are also inelastic and characterized by the same value of $\alpha$. Note that the same parameters $\mu_{r}$ and $\alpha$ are used both for the intruder particle and the surrounding smaller particles. Further details of our computer simulation are similar to those found in the literature, e.g., [25,26].

The simulation results for the migration effects are plotted black in Fig. 2. As can be seen, the simplified simulation model neglecting the detailed kinematics of rolling spheres captures all physical properties of the ex-

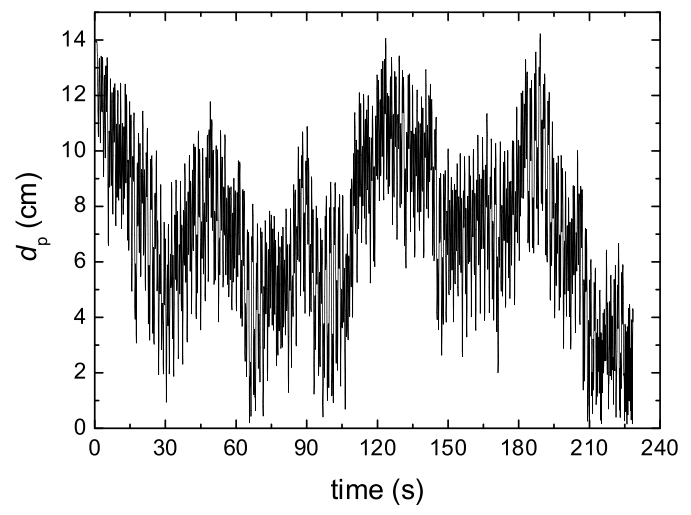

FIG. 4. Temporal evolution of the radial distance $d_{\mathrm{p}}$ from the center of the swirling dish for a $10 \mathrm{~mm}$ steel ball in 1400 glass beads (filling fraction $\mu=0.60$ ), showing no tendency for supporting any segregation effect. Parameters: $A=1.91 \mathrm{~cm}$, $f=1.67 \mathrm{~Hz}$. periments. It is able to predict the existence, the characteristic time, and the fluctuation properties of directed migration; i.e., it catches the essence of the migration effects.

However, the effects described so far do not happen for arbitrary values of the shaker amplitude and filling fraction. For instance, if the filling fraction is reduced below $\mu=0.60$ for an amplitude of $A=1.91 \mathrm{~cm}$ no clear migration trend was observed. This is illustrated in Fig. 4 where the intruder steel ball wanders through all regions of the dish.

Since this scenario is influenced by the two parameters amplitude $A$ and filling fraction $\mu$ we explored this dependence further. By increasing the filling fraction $\mu$ for several amplitudes $A$ ranging from $A=0.91 \mathrm{~cm}$ to $A=$ $2.22 \mathrm{~cm}$ (for higher amplitudes the existence of a monolayer is not granted) we were able to delimit the regions of the phase space diagram where directed migration takes place. They are shown in Fig. 5. The region marked in green corresponds to the migration of the polypropylene sphere towards the boundary. Outside this region the polypropylene sphere does not show any migration trend. In contrast, the red region where the migration of the steel ball towards the center occurs is shifted to higher filling fractions. Finally, we would like to stress that these phase diagrams are not specific for polypropylene and steel. In fact, all materials used in Fig. 1 with the same migration tendency are characterized by the same phase diagram within our experimental resolution.

Finally, the dependence of these transport phenomena on the internal parameters (size and material density) of the particles was put to a thorough experimental test with spherical beads made of 8 differently dense materials from wood $\left(\rho=0.65 \mathrm{~g} \mathrm{~cm}^{-3}\right)$ to bronze $\left(\rho=8.9 \mathrm{~g} \mathrm{~cm}^{-3}\right)$ at various diameters between 2 and $22 \mathrm{~mm}$. We prepared 60 combinations out of which 31 showed segregation to the boundary and 22 segregation towards the center. For some particle combinations an indifferent state was stable. An

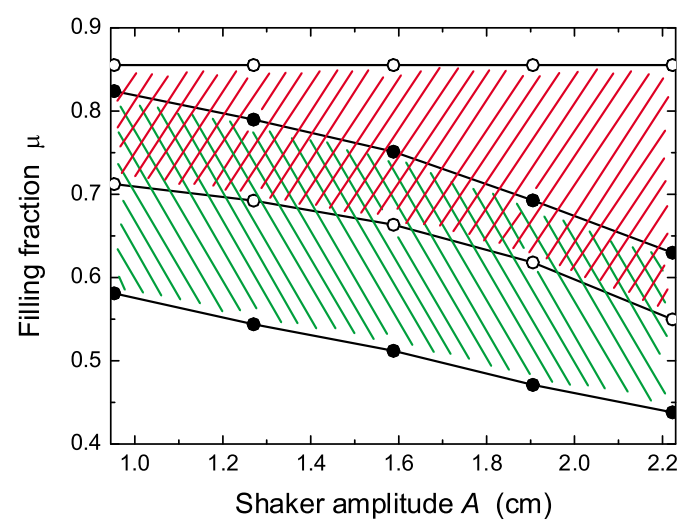

FIG. 5 (color). Phase diagrams of the critical filling fraction $\mu_{c}$ vs shaker amplitude $A$ for two intruder particles with different material properties as specified in Fig. 2. Green region: polypropylene sphere migrating to the boundary; red: steel ball drifting to the center of the bowl. 


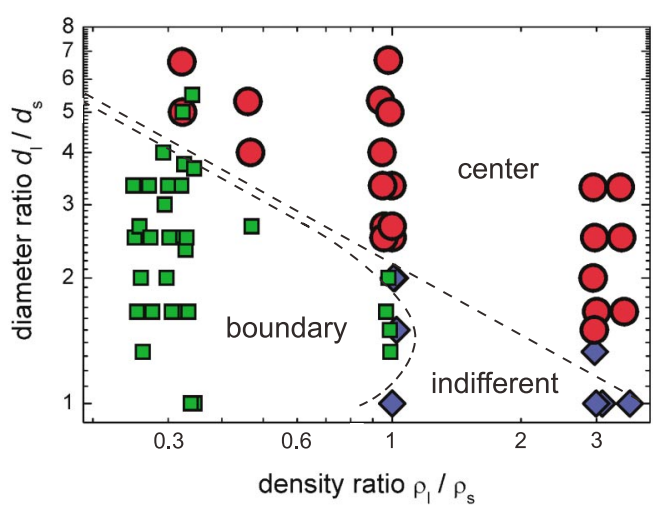

FIG. 6 (color). Phase space for particle properties. The plot shows the regimes where segregation towards the center (red circles), the boundary (green squares), or a mixed state (blue diamonds) occurs depending on the particle properties. Each symbol represents one experiment. The dashed lines indicate the numerically obtained border lines between the three different regions. Shaker amplitude $A=1.91 \mathrm{~cm}$ and frequency $f=1.67 \mathrm{~Hz}$.

overview of the results of the experimental tests is shown in Fig. 6. The explored density-ratio regime spans over 1 order of magnitude. In this phase diagram one can distinguish three regions: the upper part represents the migration towards the center, the lower left one to the boundary, and the low right corner shows the region where no migration could be observed. In order to delimit these regions we explored the phase space further by performing numerical simulations. The border lines between these regions are plotted together with the experimental data. They are determined by interpolation of numerical runs performed on a discrete grid in the two-dimensional parameter space.

To conclude, we have quantitatively characterized the temporal evolution of a horizontal analogue of the Brazilnut effect and its reverse: a heavy particle migrates to the center, while a light one moves towards the boundary of the swirling circular container. We were able to model this effect by simulating a simplified dynamics of the rolling and colliding spheres. Based on the comparison between experiment and theory we can claim that neither gravity, nor interstitial air, nor surface properties of the spheres, etc., are relevant for the migration. Thus we conclude that the density gradient along the radial direction (high density in the center and low density near the boundary) drives the effects. The more mobile light particles move into dilute areas while heavy ones with large inertia are dragged to dense regions. This is strikingly similar to the usual vertical Brazil-nut effect and its reverse.

This collaboration was made possible by Deutsche Forschungsgemeinschaft through the GRK 698.
R. B. acknowledges support by Secretaría de Estado de Educación y Universidades (Spain) and Project No. FIS2004-271.

[1] R. Lagal, Gold Panning Is Easy (Ram Publishing Company, Dallas, TX, 1992).

[2] J. C. Williams, Powder Technol. 15, 245 (1976).

[3] A. Kudrolli, Rep. Prog. Phys. 67, 209 (2004).

[4] Z.T. Chowhan, Pharmaceutical Technology 19, 56 (1995).

[5] S. Luding et al., Pharmaceutical Technology 20, 42 (1996).

[6] A. Rosato, K. J. Strandburg, F. Prinz, and R. H. Swendsen, Phys. Rev. Lett. 58, 1038 (1987).

[7] R. Jullien, P. Meakin, and A. Pavlovitch, Phys. Rev. Lett. 69, 640 (1992).

[8] J. Duran, J. Rajchenbach, and E. Clément, Phys. Rev. Lett. 70, 2431 (1993).

[9] W. Cooke, S. Warr, J. M. Huntley, and R. C. Ball, Phys. Rev. E 53, 2812 (1996).

[10] J. B. Knight, H. M. Jaeger, and S. R. Nagel, Phys. Rev. Lett. 70, 3728 (1993).

[11] M.E. Möbius, B.E. Lauderdale, S. R. Nagel, and H. M. Jaeger, Nature (London) 414, 270 (2001).

[12] T. Shinbrot and F. J. Muzzio, Phys. Rev. Lett. 81, 4365 (1998).

[13] D. C. Hong, P. V. Quinn, and S. Luding, Phys. Rev. Lett. 86, 3423 (2001).

[14] N. Shishodia and C. R. Wassgren, Phys. Rev. Lett. 87, 084302 (2001).

[15] J. A. Both and D.C. Hong, Phys. Rev. Lett. 88, 124301 (2002).

[16] A. P. J. Breu, H. M. Ensner, C. A. Kruelle, and I. Rehberg, Phys. Rev. Lett. 90, 014302 (2003).

[17] D. A. Huerta and J. C. Ruiz-Suárez, Phys. Rev. Lett. 92, 114301 (2004).

[18] Y. Nahmad-Molinari, G. Canul-Chay, and J.C. RuizSuárez, Phys. Rev. E 68, 041301 (2003).

[19] K. Liffman et al., Granular Matter 3, 205 (2001).

[20] S. Aumaître, C. A. Kruelle, and I. Rehberg, Phys. Rev. E 64, 041305 (2001).

[21] S. Aumaître, T. Schnautz, C. A. Kruelle, and I. Rehberg, Phys. Rev. Lett. 90, 114302 (2003).

[22] P. M. Reis and T. Mullin, Phys. Rev. Lett. 89, 244301 (2002).

[23] P. M. Reis, G. Ehrhardt, A. Stephenson, and T. Mullin, Europhys. Lett. 66, 357 (2004).

[24] L. Kondic, Phys. Rev. E 60, 751 (1999).

[25] S. Luding and S. McNamara, Granular Matter 1, 113 (1998).

[26] N. Brilliantov and T. Pöschel, Kinetic Theory of Granular Gases (Oxford University Press, New York, 2004); J. Comput. Phys. 34, 184 (1980). 